\title{
Incorporating Prior Biologic Information for High-Dimensional Rare Variant Association Studies
}

\author{
Melanie A. Quintana ${ }^{a} \quad$ Fredrick R. Schumacher ${ }^{\mathrm{a}}$ Graham Casey $^{\mathrm{a}}$ \\ Jonine L. Bernstein ${ }^{c} \quad$ Li Li $^{b}$ David V. Conti ${ }^{\mathrm{a}}$ \\ ${ }^{a}$ Department of Preventive Medicine, University of Southern California, Los Angeles, Calif., b Department of \\ Epidemiology and Biostatistics, Case Western Reserve University, Cleveland, Ohio, and ' ${ }^{\mathrm{D}}$ epartment of \\ Epidemiology and Biostatistics, Memorial Sloan-Kettering Cancer Center, New York, N.Y., USA
}

\section{Key Words}

Genetic association studies - Bayesian model uncertainty ·

Bayes factors · Sequence analysis · Rare variant analysis

\begin{abstract}
Background: Given the increasing scale of rare variant association studies, we introduce a method for high-dimensional studies that integrates multiple sources of data as well as allows for multiple region-specific risk indices. Methods: Our method builds upon the previous Bayesian risk index by integrating external biological variant-specific covariates to help guide the selection of associated variants and regions. Our extension also incorporates a second level of uncertainty as to which regions are associated with the outcome of interest. Results: Using a set of study-based simulations, we show that our approach leads to an increase in power to detect true associations in comparison to several commonly used alternatives. Additionally, the method provides multilevel inference at the pathway, region and variant levels. Conclusion: To demonstrate the flexibility of the method to incorporate various types of information and the applicability to high-dimensional data, we apply our method to a single region within a candidate gene study of second primary breast cancer and to multiple regions within a candidate pathway study of colon cancer.
\end{abstract}

Copyright ๑ 2013 S. Karger AG, Basel

\section{KARGER}

E-Mail karger@karger.com

www.karger.com/hhe

\section{Introduction}

Rare variant association studies have gained a tremendous amount of popularity and there has been a concurrent surge in the development of methods to detect associations between rare variants and complex disease outcomes. The majority of these methodological developments center around the idea of collapsing variants within a single region to test the collective frequency or weighted frequency of the variants within the cases and controls [1-3]. While these methods are powerful in detecting associated regions, they do not allow inference at the variant level and there can be a substantial loss in power when the effects of the rare variants are mixed (both risk and protective). Moreover, many methods that aggregate all variants in region without a model selection procedure have a substantial loss of power as the number of null variants increases (holding the number of associated variants constant) and the noise overwhelms the signal. More recent work has expanded upon these basic collapsing methods to formally incorporate uncertainty into the variants that enter the risk index and to account for the direction of effect of the variants [4-7]. There has also been an emergence of methods that focus on testing the hypothesis that there is an increase or decrease in the probability of some of the mutations being found in the affected individuals

David V. Conti, PhD

Division of Biostatistics, University of Southern California 2001 N. Soto Street, 202S

Los Angeles, CA 90089 (USA)

E-Mail dconti@usc.edu 
by computing a test statistic that is based on determining if the mixture distribution of probabilities under the alternative hypothesis has variance. This test on the variance of the mixture distribution bypasses the need to make an assumption on the direction of the effect of each variant $[8,9]$. As a unified approach, the Bayesian risk index (BRI) [5] formally incorporates uncertainty of both the inclusion of variants and the direction of effect via Bayesian model uncertainty (BMU) techniques and it allows for intuitive multi-level inference at the region and variant level. This multi-level inference of BRI provides the practitioner with the ability to pinpoint specific variants that are driving a regional association.

To date, most statistical methods developed for rare variant association studies have focused on demonstrating power in small-scale studies involving a single region. As large-scale chip arrays and sequencing technologies become more efficient and cost effective, the scale of studies involving rare variants has begun to drastically increase with a shift from candidate regions or gene studies to pathway and whole-exome studies involving millions of variants. Thus, there is a need of more powerful tools to analyze large-scale rare variant studies involving multiple regions across the genome. To accommodate multiple regions, one possible solution is to perform independent tests across each region using the current collapsing methods. However, this approach leads to uncertainty in determining an appropriate significance threshold accounting for the multiple tests within and across regions and the approach does not borrow strength across regions. Within studies involving common variants, it has been shown that modeling a multi-variate genetic profile via BMU methods leads to an increase in power to detect true associations over marginal tests [10]. Thus, it may be more appropriate to bypass the assumption of conditional independence of the regions and model the outcome as a function of a multi-regional genetic profile in order to borrow strength across regions. With this in mind, our integrative BRI allows for multiple region-specific risk indices within each model. As in BRI, we formally incorporate uncertainty into the variants that are included within the region-specific risk index as well as the direction of effect for each variant. However, we also introduce a second level of uncertainty as to which region-specific risk indices are included in the model. This second level incorporates prior information and builds upon a recent extension of BMU that integrates external biological covariates into the probability that any variant is associated (iBMU). This approach has been shown to lead to an increase in power and a more efficient model search over other commonly used variable selection techniques [unpubl. data]. For rare variant analysis, we extend the current BRI method by incorporating external variant-specific covariates into the probability that any variant is included within a risk index in order to gain power in detecting rare variation within large-scale studies.

The remainder of the paper is organized as follows: the Methods section gives a brief review of the BMU framework and more specifically the BRI. We then describe the extension of BRI to incorporate multiple regions and integrate external biological knowledge, denoted as iBRI. In this section, we also describe a set of single region and a set of multiple region simulations which we will use to assess the power of iBRI compared to several alternative methods. The next section provides the results of the simulation study as well as results of two applications of iBRI to detecting rare variants within a candidate gene study of second primary breast cancer and a candidate pathway study of colon cancer using the Illumina exome chip. In the final section, we end with a discussion of our novel method as well as future directions.

\section{Methods}

\section{BRI Overview}

Detailed descriptions of the general BMU framework have been described previously $[11,12]$. For rare variant analysis in genetic association studies, we assume that for a set of $n$ individuals we have: (1) an $n$-dimensional binary outcome vector $\mathbf{Y}$ that represents an individual's disease status, (2) a set of $p$ genotypes within a $(n \times p)$-dimensional matrix $\mathbf{G}$, where $G_{i v}=0,1,2$, for the number of copies of the minor allele measured for individual $i$ at variant $v$, and (3) a set of $q$ covariates within a $(n \times q)$-dimensional matrix $\mathbf{Z}$ included in all models. These covariates include variables such as age and sex, and variables used to control for potential confounding by population stratification. Within the BMU framework, we consider all models $\mathbf{M}_{\gamma} \in \mathbf{M}$, defined by a distinct subset of $p$ genetic variants and including all $q$ adjustment variables in each model. In particular, each model $\mathbf{M}_{\gamma}$ is indexed by a $p$-dimensional indicator vector $\gamma$, where $\gamma_{v}=1$ if variant $v$ is included in model $\mathbf{M}_{\gamma}$ and $\gamma_{v}=0$ if $v$ is not included in model $\mathbf{M}_{\gamma}$. Within BRI [5], we extend each model in the BMU framework to also indicate the direction of effect for each variant if included, so that if $\gamma_{v}=1$, variant $v$ is included as a risk factor, and if $\gamma_{v}=-1$, variant $v$ is included as a protective factor.

Then, given any model $\mathbf{M}_{\gamma}$, we define a risk index as the collective frequency of the variants in model $\mathbf{M}_{\gamma}$ that is of the form:

$$
\mathbf{X}_{i \gamma}=\sum_{v=1}^{p} \gamma_{v} G_{i v} .
$$

We can then relate the outcome to the risk index based on the logistic regression formula:

$$
\mathbf{M}_{\gamma}: \operatorname{logit}\left(Y_{i}=1\right)=\beta_{0}+\beta \mathbf{Z}_{i}+\beta_{\gamma} \mathbf{X}_{\mathbf{i} \gamma} .
$$


Within this model specification, we note that for each risk index, $\mathbf{X}_{\gamma}$, we have a single effect, $\beta_{\gamma}$, for the group of variants included in the model which we will refer to as the rare variable load.

\section{Multiple Regions within BRI}

As described in Quintana et al. [5], the BRI framework is based on modeling the relationship of the outcome variable and a single risk index that is formed by looking at the collective frequency of a subset of variants. This framework works best when the total number of variants of interest is relatively small and within a single region. To extend this approach, we assume that we have genotype data on a set of $p$ genetic variants that belong to a set of $p_{R}$ regions and we wish to model the outcome variable using multi-regional genetic profile. In particular, for each model $\mathbf{M}_{\gamma}$, we wish to incorporate a risk index for each region $R_{j}$ defined as:

$$
\mathbf{X}_{i R_{j} \gamma}=\sum_{v \in R_{j}} \gamma_{v} G_{i v} .
$$

We then relate the outcome to the risk indices for each region based on the logistic regression formula:

$$
\mathbf{M}_{\gamma}: \operatorname{logit}\left(Y_{i}=1\right)=\beta_{0}+\beta \mathbf{Z}_{i}+\sum_{j=1}^{p_{R}} \beta_{\mathbf{R}_{\mathbf{j}} \gamma} \mathbf{X}_{i R_{j} \gamma} .
$$

Here, $\beta_{R_{j} \gamma}$ is the model-specific rare variant load for region $R_{j}$. We note that when $\gamma_{v}=0$ for all variants in region $\mathrm{R}_{j}$, the region-specific risk index is not included in our model. Thus, our extension of BRI for multiple regions introduces a second level of uncertainty on the regions associated with the outcome of interest as well as the variants included in each region-specific risk index.

\section{Integrative Bayesian Risk Index}

In addition, we wish to extend the current BRI framework to integrate external variant-specific biological information. We denote our integrative extension as iBRI. In particular, we integrate a set of $c$ variant-specific covariates specified within a $(p \times c)$-dimensional covariate matrix $\mathbf{W}$ into the estimation of marginal inclusion probabilities by introducing a second-stage regression on the probability that any variant is associated. Specifically, we define the probability that any variant is associated as a function of the variant-specific covariates using a probit model:

$$
\operatorname{probit}\left(p\left(\gamma_{v} \neq 0 \mid \alpha_{0}, \alpha\right)\right)=\alpha_{0}+\alpha \mathbf{W}_{v} .
$$

Here, $\alpha$ is a $c$-dimensional vector of regression coefficients quantifying the increase or decrease in probability of association of each variant given the variant-specific covariate and $\alpha_{0}$ specifies a baseline probability of association common to all variants. Similar to Wilson et al. [10], we define the baseline probability to incorporate an implicit multiplicity correction, in that the prior probability of at least one variant being associated remains constant as the total number of variants of interest increases. That is, $\alpha_{0}=\Phi^{-1}\left(2^{-1 / p}\right)$ where $\Phi^{-1}()$ is the inverse of the normal cdf.

\section{Posterior Multi-Level Inference}

Given any model $\mathbf{M}_{\gamma} \in \mathbf{M}$, we can quantify the evidence that the data supports the model via posterior model probabilities defined as:

$$
p\left(\mathbf{M}_{\gamma} \mid \mathbf{Y}\right)=\frac{p\left(\mathbf{Y} \mid \mathbf{M}_{\gamma}\right) p\left(\mathbf{M}_{\gamma}\right)}{\sum_{\mathbf{M}_{\gamma} \in \mathbf{M}} p\left(\mathbf{Y} \mid \mathbf{M}_{\gamma}\right) p\left(\mathbf{M}_{\gamma}\right)}
$$

where $p\left(\mathrm{Y} \mid \mathrm{M}_{\gamma}\right)$ is the marginal likelihood of each model after integrating our model-specific parameters, $\theta_{\gamma}$, and $p\left(\mathrm{M}_{\gamma}\right)$ is the prior probability of model $\mathrm{M}_{\gamma}$. Wilson et al. [10] provide a detailed description of how to approximate the marginal likelihood and specify the prior model probability to incorporate an implicit multiplicity correction. This specification allows the prior probability at baseline of at least one variant being associated to remain constant as the total number of variants of interest increases.

We can use the posterior model probabilities to provide intuitive multi-level inference for any predefined set of the predictor variables of interest. In particular, for some set of variants, $v \in \mathbf{S}$, we can quantify the evidence that at least one variant within the set is associated via set-specific posterior probabilities:

$$
p\left(\Gamma_{S}=1 \mid Y\right)=\sum_{\mathbf{M}_{\gamma} \in \mathbf{M}: \Gamma_{S}=1} p\left(\mathbf{M}_{\gamma} \mid \mathbf{Y}\right),
$$

where $\Gamma_{S}=1$, if at least one variant within set $\mathbf{S}$ is in model $\mathbf{M}_{\gamma}$. Thus, the posterior probability for any set is simply the sum of the posterior model probabilities for every model that includes at least one variant within the set. In genome-wide rare variant studies, some examples of multi-level quantities of interest are: (1) the genome-wide global posterior probability that at least one variant is associated (calculated as the sum of the posterior model probabilities of all of the non-null models), (2) the gene-level posterior probability that at least one variant is associated within a gene (calculated as the sum of the posterior model probabilities of all of the models that include a variant within a specific gene), and (3) marginal posterior probabilities that any particular variant $v$ is associated (calculated as the sum of the posterior model probabilities of all models that include variant $v$ ). Finally, given any multi-level posterior probability, $p\left(\Gamma_{S}=1 \mid \mathbf{Y}\right)$, we can also calculate the multilevel Bayes factors (BF) as the posterior odds that at least one variant within the set is associated divided by the prior odds:

$$
\mathrm{BF}\left[\Gamma_{S}=1: \Gamma_{S}=0\right]=\frac{p\left(\Gamma_{S}=1 \mid Y\right)}{p\left(\Gamma_{S}=0 \mid Y\right)} \div \frac{p\left(\Gamma_{S}=1\right)}{p\left(\Gamma_{S}=0\right)} \text {. }
$$

\section{Posterior Computation}

To compute posterior model probabilities and estimate the regression coefficients of the second-stage model on the marginal inclusion probabilities, we iterate between a Metropolis Hastings $(\mathrm{MH})$ algorithm to sample models $\mathbf{M}_{y} \in \mathbf{M}$ and a Gibbs sampling algorithm to sample the second-stage regression coefficients $\alpha$. Within the MH algorithm, new models are proposed based on randomly selecting one variant and changing its status within the current model. Within the Gibbs algorithm, full conditionals are calculated to sample from the posterior distribution of $\alpha$ given the current model and model-specific parameters. By iterating between the $\mathrm{MH}$ and Gibbs algorithms, we can obtain a sample of models from the model space as well as a sample from the posterior distribution of $\alpha$ which is used to approximate the posterior model probabilities as well as the multi-level posterior summaries. In particular, the posterior model probabilities are renormalized over the sum of sampled models and the prior model probabilities, $p\left(\mathbf{M}_{\gamma}\right)$, are approximated by using the Monte Carlo estimates of the inclusion probabilities, $p\left(\gamma_{v} \neq 0\right)$, given the sampled values of $\alpha$. The sampling algorithms and calculation of all of the posterior summaries are available in the Bayesian variant selection (BVS) R package on CRAN [13]. 


\section{Simulation Study}

We wish to assess the power of iBRI compared to several commonly used alternatives on a set of single region simulations as well as a set of multiple region simulations. In particular, we compare our method to the original non-integrative BRI of Quintana et al. [5], the weighted sum statistic of Madsen and Browning [3], the C-alpha method of Neale et al. [8], and the comprehensive step-up approach of Hoffmann et al. [4] within a single and multiple regions. For the simulations involving multiple regions, we compute independent tests of each region under the BRI, weighted sum, Calpha and comprehensive step-up approaches.

We created a set of 1,000 simulations that were comprised of a single region of variants based on the WECARE Study (described in BRCA1 Results). Each single region simulation is based on the available genetic data of 134 rare variants within BRCA1 for 1,912 individuals as part of the WECARE Study. We also created a set of 1,000 multiple region simulations. Each multiple region simulation is based on exome-array data from The Kentucky Colorectal Cancer Study (KY - described in Exome Analysis of DNA Repair Pathway Results) including 114 genes within the DNA repair pathway and comprised of 473 rare variants for 1,356 individuals. The 114 genes play a role within 10 unique DNA repair sub-pathways. For each simulation within both the single and multiple region simulation sets:

(1) We define variant-specific covariates. For the single region simulations, variant-specific covariates are defined within a $(134 \times 4)$-dimensional matrix $\mathbf{W}$ indicating the effect type of each variant (intervening sequence (IVS), synonymous, missense, truncation). For the multiple region simulations, variant-specific covariates are defined within a $(473 \times 10)$-dimensional matrix $\mathbf{W}$ indicating the DNA repair sub-pathway that each variant is involved in.

(2) We randomly select one of these variant-specific covariates and sample an $a$ within $\{0,1,2,3\}$ for that covariate (all of the other covariates are assumed to have an a level of 0 ). Marginal probabilities of association are then calculated for each rare variant based on the assigned $\alpha$ levels.

(3) We select between 0:10 causal rare variants based on the marginal probabilities.

(4) We randomly select a $\log (\mathrm{OR})$ for all causal rare variants within $\{0.5,1,1.5,2,2.5\}$.

(5) We simulate each individual's case/control status based on the selected causal variants and $\log (\mathrm{OR})$.

We note that when the a level for the associated variant-specific covariate is selected to be 0 , the casual variants within the simulation are independent from all variant-specific covariates and the simulation is referred to as a non-informative simulation. When the a level for the associated variant-specific covariate is greater than 0 , we refer to the simulation as informative.

\section{Results}

\section{Simulation Results}

We ran the iBRI and rare variant alternative analyses on the set of 1,000 single region WECARE Study-based simulations as well as the set of 1,000 multiple region KY study-based simulations. In particular, we compare the power to detect regional associations of the rare variants using iBRI with BRI, the weighted sum approach, C-alpha, and the comprehensive step-up approach. We also compare the power to detect marginal associations of the rare variants within the simulated data under iBRI and BRI. We plot regional and marginal ROC curves for each method for the single region WECARE Study-based simulations and the multiple region KY study-based simulations in figure 1 and 2, respectively. Specifically, in panels $\mathrm{a}$ and $\mathrm{b}$ of both figure 1 and 2 , the regional true positive rate (TPR) is plotted against the regional false positive rate (FPR) as we vary the regional $\mathrm{BF}$ threshold for the $i B R I$ and BRI approaches and the regional $p$ value threshold for the weighted sum, C-alpha and comprehensive step-up approaches. In panel a, we calculate regional TPR and FPR within all non-informative simulations and in panel $b$, we calculate the same quantities within all informative simulations.

Within regions that demonstrate strong evidence of an association amongst the rare variants, we compare the power to pinpoint which of the rare variants are most likely driving the regional associations. In particular, in panels $\mathrm{c}$ and $\mathrm{d}$ of both figure 1 and 2, the marginal TPR is plotted against the marginal FPR as the marginal $\mathrm{BF}$ threshold varies under the iBRI and BRI approaches. In panel $c$, we calculate the marginal TPR and FPR within all non-informative simulations and in panel $\mathrm{d}$, we calculate the same quantities within all informative simulations.

Within figures 1 and 2 we see that iBRI and BRI have slightly higher power to detect regional associations over the alternative methods within our single and multiple region simulations. Also, when the variant-specific covariates are truly informative with regards to the casual variants, iBRI shows an increase in power to detect associated regions over the alternatives (fig. $1 \mathrm{~b}, 2 \mathrm{~b}$ ) and shows an increase in power to detect associated variants over the basic BRI (fig. 1d, 2d). This potential increase does not come with a corresponding drastic loss in power to detect associated regions and variants of iBRI when the variantspecific covariates are truly uninformative (fig. 1a, c, $2 \mathrm{a}, \mathrm{c})$.

Given the tremendous size of the model space when we are analyzing multiple regions simultaneously with iBRI, we also assess the degree to which the integration of informative variant-specific covariates can influence the speed of convergence of the model search algorithm. In particular in figure 3 , we plot the number of iteration until a truly causal variant (panel a) and non-causal variant (panel b) is sampled as a function of the known simulated value of $\alpha$. As the variant-specific covariates become more 
Fig. 1. ROC curves for single region WECARE Study-based simulations. Regional FPR are plotted against regional TPR as the regional $\mathrm{BF}$ threshold varies for iBRI and $\mathrm{BRI}$ and as the regional $\mathrm{p}$ value thresholds vary for the weighted sum, C-alpha, and comprehensive (Comp.) step-up approaches $(\mathbf{a}, \mathbf{b})$. Marginal FPR are plotted against marginal TPR as the marginal BF threshold varies for iBRI and BRI (c, d). Panels $\mathbf{a}$ and $\mathbf{c}$ are across all non-informative simulations and panels $\mathbf{b}$ and $\mathbf{d}$ are across all informative simulations.

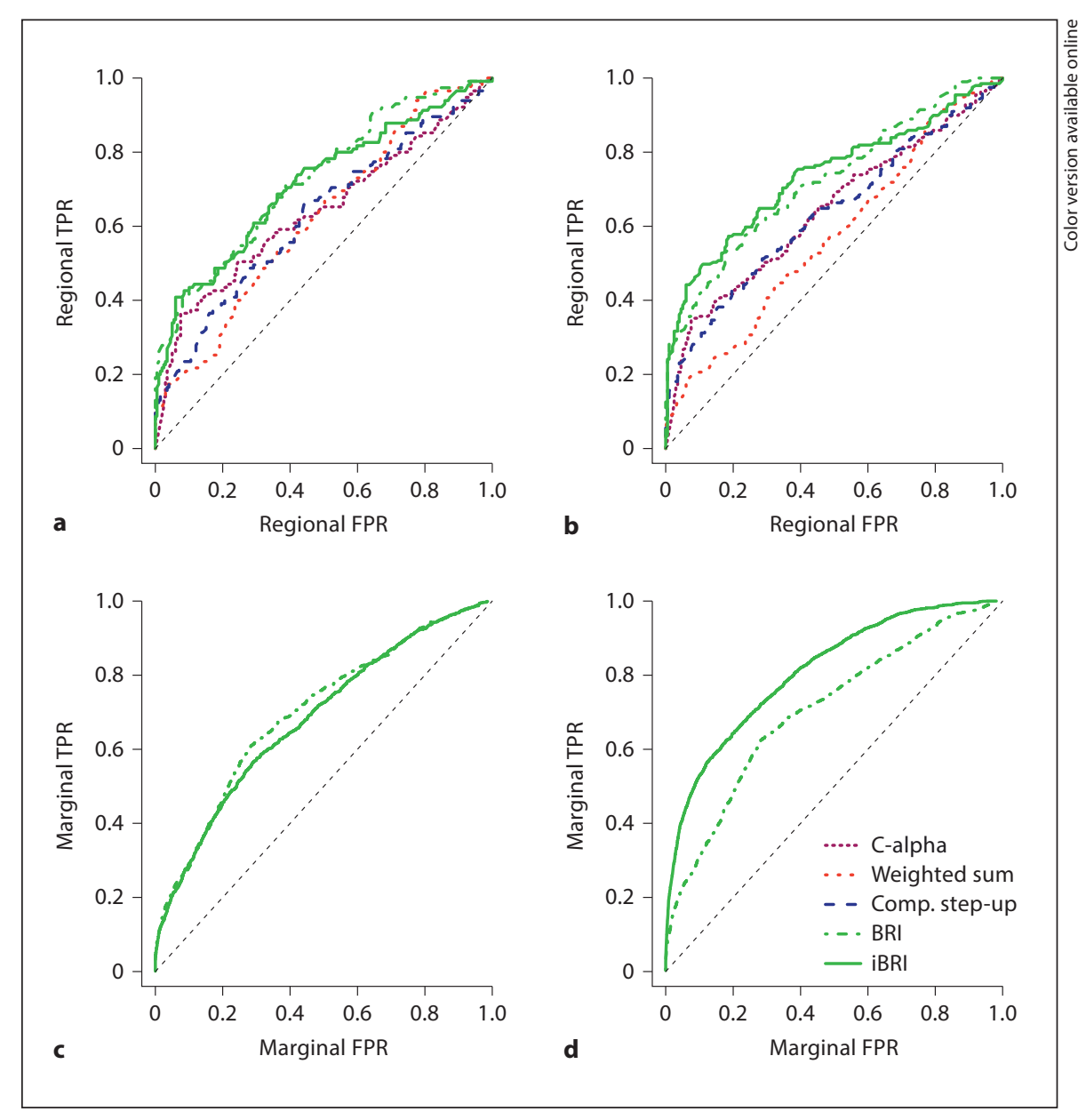

informative, there is a decrease in the mean number of iterations needed for a causal variant to be sampled. Additionally, there is no increase in the mean number of iterations needed to sample non-causal variants. Thus, the integration of external biological covariates with iBRI leads to a more efficient model search algorithm when these covariates are truly informative with regard to the causal variants.

\section{BRCA1 Results}

The WECARE (Women's Environmental Cancer and Radiation Epidemiology) Study is a population-based case-control study designed to investigate genetic risk factors of second primary breast cancer. For a more detailed description of the study, see Bernstein et al. [14]. The study includes 640 non-Hispanic women with contralateral breast cancer (cases) and 1,272 women with unilateral breast cancer (controls), individually matched based on age at and year of primary diagnosis, race, and reporting registry. For all participants, the complete coding sequences of $B R C A 1$ (5,589 bp, split into 22 coding exons) and $B R C A 2$ (10,254 bp and 26 coding exons) were screened for variations by denaturing high-performance liquid chromatography, using leukocyte genomic DNA as a template, identifying a large number of both common and rare variants [15-17]. To demonstrate the use of iBRI within a single region of a candidate pathway study, we analyze all self-identified white individuals with a logistic regression adjusted by age to investigate a subset of 134 rare $B R C A 1$ variants (minor allele frequency (MAF) between 0.0003 and 0.05 ) and integrate variant-specific biological covariates of whether or not a variant is known as being deleterious and the effect type of each variant (IVS, synonymous, missense, truncation) into the analysis to help guide variant selection. Thus, for the WECARE Study analysis, $\mathbf{W}$ is a $(134 \times 5)$-dimensional matrix.

By using our novel iBRI approach within BRCA1 and integrating external variant-specific biological covariates 
Fig. 2. ROC curves for multiple region exome study-(KY) based simulations. Regional FPR are plotted against regional TPR as the regional BF threshold varies for iBRI and BRI and as the regional $p$ value thresholds vary for the weighted sum, Calpha, and comprehensive (Comp.) stepup approaches $(\mathbf{a}, \mathbf{b})$. Marginal FPR are plotted against marginal TPR as the marginal $\mathrm{BF}$ threshold varies for $\mathrm{BRRI}$ and $\mathrm{BRI}$ (c, d). Panels a and $\mathbf{c}$ are across all non-informative simulations and panels $\mathbf{b}$ and $\mathbf{d}$ are across all informative simulations.
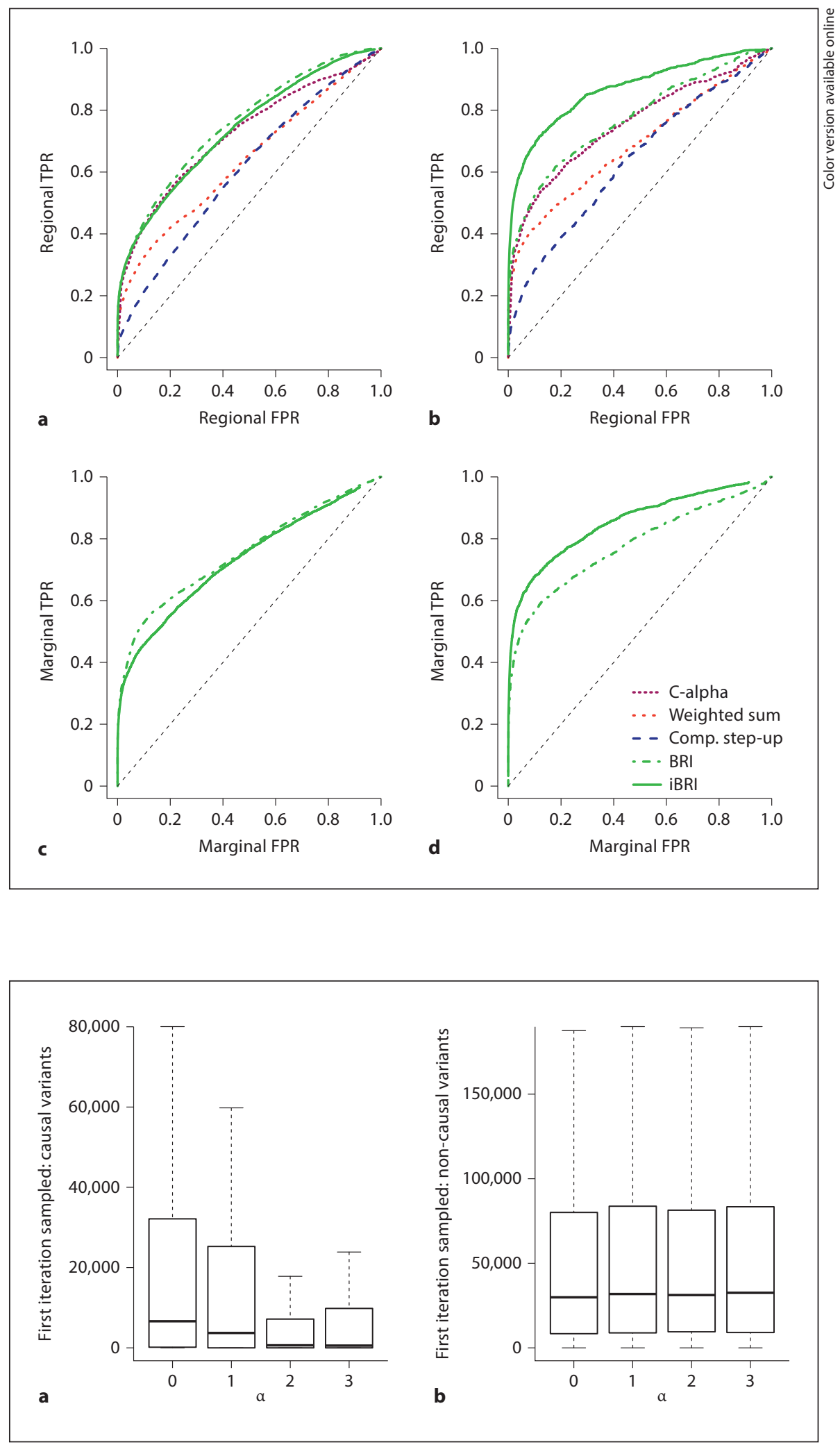

Fig. 3. Sampling causal and non-causal predictors in multiple region exome study(KY) based simulations. We plot the number of iterations under iBMU until the first acceptance of the causal (a) and non-causal (b) variants as a function of the known simulated value of $\alpha$. 
Table 1. BRCA1: top 10 variants found using iBRI

\begin{tabular}{|c|c|c|c|c|c|c|}
\hline \multirow[t]{2}{*}{ Effect } & \multirow[t]{2}{*}{ Variant } & \multirow[t]{2}{*}{ Exon } & \multirow[t]{2}{*}{ Deleterious } & \multirow[t]{2}{*}{ Cases/controls } & \multicolumn{2}{|c|}{ Marginal BF } \\
\hline & & & & & iBRI & BRI \\
\hline \multirow{6}{*}{$\begin{array}{l}\text { Truncation } \\
(\mathrm{BF}=7.4 \mathrm{e}+08)\end{array}$} & $\mathrm{V} 2$ & 2 & Yes & $9 / 4$ & $3,667.3$ & 42.4 \\
\hline & V122 & 11 & Yes & $3 / 0$ & $1,927.0$ & 32.1 \\
\hline & V52 & 11 & Yes & $2 / 0$ & 692.5 & 13.5 \\
\hline & $\mathrm{V} 3$ & 2 & Yes & $2 / 0$ & 688.7 & 13.9 \\
\hline & V112 & 11 & Yes & $2 / 0$ & 683.7 & 12.8 \\
\hline & V106 & 11 & Yes & $2 / 0$ & 662.4 & 13.3 \\
\hline Missense & V13 & 5 & Yes & $8 / 2$ & $15,690.9$ & 124.4 \\
\hline$(\mathrm{BF}=4.1 \mathrm{e}+03)$ & V8 & 3 & Yes & $2 / 0$ & 413.9 & 13.3 \\
\hline IVS $(\mathrm{BF}=7.0)$ & V137 & 12 & No & $2 / 0$ & 26.3 & 13.0 \\
\hline Synonymous $(\mathrm{BF}=1.0)$ & V7 & 3 & No & $2 / 0$ & 5.1 & 9.7 \\
\hline
\end{tabular}

Variants are organized based on the effect type of each variant. The effect type BFs are reported under each effect.

of whether a variant is known as being deleterious and the effect type of each variant, we find a regional $\mathrm{BF}$ of $4.7 \mathrm{e}+10$, giving extremely strong evidence that at least one variant within BRCA1 is associated with second primary breast cancer. Using the non-integrative BRI, we find a modest regional BF of 29.3. Given the extremely strong evidence of an association within $B R C A 1$, we are interested in pinpointing the variants most likely driving the association. In table 1 , we provide information on the top 10 variants found using iBRI. In particular, variants are organized based on their effect type and each effect type is ordered based on its BF giving evidence that at least one variant with the corresponding effect type is associated. For each variant, we also report: (1) the exon the variant is located in, (2) if a variant is known as being deleterious, (3) the count of cases and controls that have the variant, (4) the marginal BF under iBRI, and (5) the marginal $\mathrm{BF}$ under BRI. The variants which are identified as either with a truncation or missense effect are most likely associated with second primary breast cancer. By integrating data on each variant's effect type and if a variant is known as being deleterious, we find that the variants with a truncation or missense effect, as well as variants that are known as being deleterious, have a great increase in marginal BFs using iBRI over the non-integrative BRI. This is reflected in an elevated estimate for a corresponding to the 'deleterious' covariate of 2.39 (95\% credible interval (CI) 1.09-3.61) and for a corresponding to the 'truncation' covariate of 0.55 (95\% CI -0.77 to 1.88$)$. In contrast, we do not see a dramatic increase in evidence for the two variants defined as synonymous or IVS effect type and they are not known as being deleterious variants. We also note that marginal BFs under the iBRI and BRI for variants V52, V3, V112 and V106 are slightly different although the data for each is identical (counts in cases and controls and predictor-level covariates). In part, this reflects the uncertainty in the estimation of each BF. In addition, this also reflects that each marginal $\mathrm{BF}$ is calculated conditional upon all other markers in the analysis. Thus, if two variants are correlated or found in the same individual, it is unlikely that they will co-occur within the same top model, since the added information from the additional variant is reduced. This will cause a dilution in the marginal $\mathrm{BF}$ of the less likely marker driving the association.

\section{Exome Analysis of DNA Repair Pathway Results}

The KY study was initiated in July 2003 through the University of Kentucky Cancer Center [18]. A web-based reporting system implemented by the Kentucky Cancer Registry in 2003 has facilitated rapid report of cases statewide, with approximately $76.8 \%$ of all cases reported to the registry within 6 months of diagnosis. Cases $(>21$ years) diagnosed with histologically confirmed colon cancer and entered into the registry within 6 months of their diagnoses are invited to join the study. Unrelated controls are recruited through random digit dialing and are frequency matched to the cases by age ( \pm 5 years), gen- 
der, and race. Excluded from the study are those individuals who have been diagnosed with colon cancer because of known hereditary forms of colon cancer or polyposis such as familial adenomatous polyposis (FAP), hereditary non-polyposis colorectal cancer (HNPCC), PeutzJeghers, and Cowden disease. Currently there are more than 1,040 incident population-based cases of colorectal cancer and 1,750 population-based controls fully recruited, with comprehensive epidemiologic data, pathology data, and DNA from cases and controls. 338 cases with positive family history, defined as having a first- or second-degree relative with colon cancer, and 1,018 controls with negative family history from the KY study were genotyped using the Illimina Infinium Human Exome array (genome.sph.umich.edu/wiki/Exome_Chip_Design). The array was constructed from exome sequencing 10,789 exomes, thus capturing $60 \%$ of non-synonymous SNPs with an allele frequency of $1 / 7,000,80 \%$ of SNPs with an allele frequency of $1 / 5,000$ and $>99 \%$ of SNPs with an allele frequency of $1 / 1,000$. While the overall array includes 65,613 rare variants (MAF $<0.01$ ) across 15,010 gene regions, to demonstrate the use of iBRI within multiple regions of a candidate pathway study, we investigate a subset of 473 rare variants (MAF between 0.0007 and 0.01 ) in 151 DNA repair pathway genes (114 with observed variants, see online supplementary table 1; www. karger.com/doi/10.1159/000346021 for more details). The first-stage model includes sex, age and the first four principle components from an investigation of population structure. We integrate variant-specific biological covariates that indicate the specific DNA repair pathway that the variant is involved in into the analysis to help guide variant selection. For the KY exome analysis, $\mathbf{W}$ is a $473 \times 10$ matrix. See online supplementary table 1 for variant, gene and subpathway details.

Using our novel iBRI approach within the 114 unique gene regions of the DNA repair pathway we find a global $\mathrm{BF}$ of 13.0 giving strong evidence that at least one variant within the entire analysis is associated. This global summary is not available with the other approaches of testing each region individually, using the current collapsing methods of BRI, C-alpha, comprehensive step-up, and weighted sum. Given strong evidence of a global association within the study, we are interested in pinpointing the likely regions (or genes) that are driving the association. With this in mind, figure 4 plots the inclusion of the top 10 regions found using iBRI within the top 25 models. On the $\mathrm{x}$-axis, we order the top regions based on the regional BFs (reported on the right margin) and on the y-axis, we have the top models found ordered based on posterior

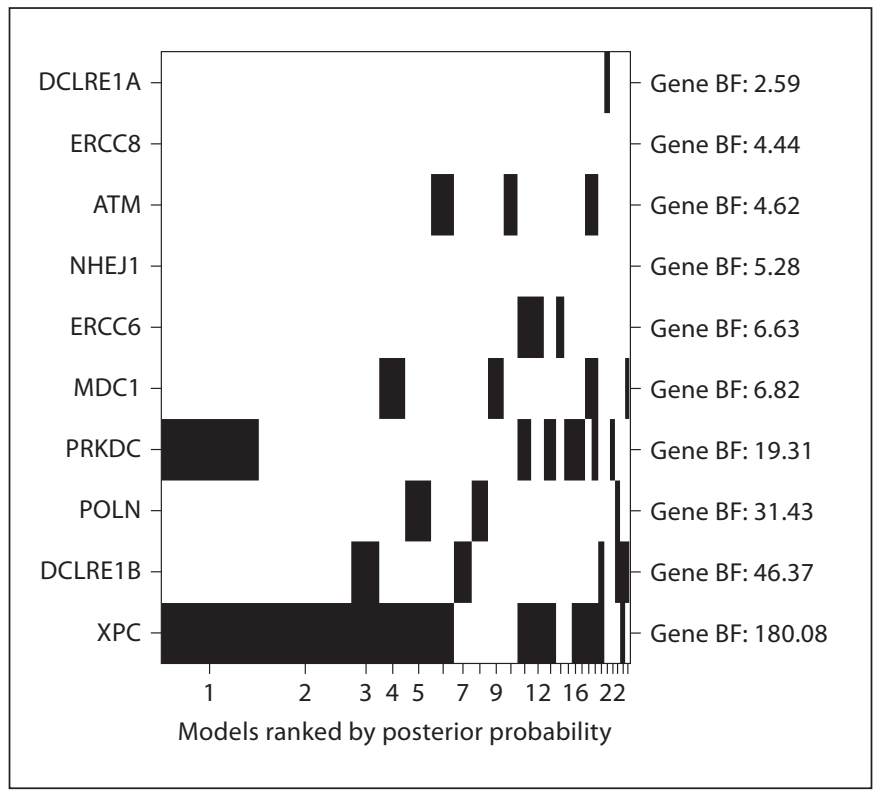

Fig. 4. Top model inclusions for top regions in DNA repair pathway. We plot the top regions (or genes) included in the top 25 models found under the iBRI approach. The inclusions/exclusions of the top 10 regions are plotted in the rows and the rows are ordered based on each regional BF (reported on the right axis). The regions included/excluded within each of the top 25 models are plotted in the columns. These columns have widths proportional to and are ordered based on the posterior probability of the corresponding model.

model probabilities. Each column represents the inclusions/exclusions of the top 10 regions within the respective model (a region is defined as being included in a model, if at least one variant within the region is included in the model) and the width of each column is proportional to the posterior model probability of the top model. We can see in figure 4 that the top model includes at least one variant within gene $X P C$ and gene PRKDC. In fact, many of the top models include at least one variant within multiple regions. Thus, it is likely that modeling the outcome as a multi-regional genetic profile will give us added power over testing each region individually. Table 2 reports more information on the top 10 genes found using the iBRI approach. Within the table, the genes are organized based on the specific DNA repair pathway that the gene is involved in and the pathway BFs that quantify the evidence that at least one variant within the specific DNA repair pathway is associated are reported under each pathway. Next to each gene we report: (1) the gene $\mathrm{BF}$ found using iBRI, (2) the most likely associated variant within the gene determined based on marginal BF, (3) the 
Table 2. DNA repair pathway: top 10 genes found using iBRI

\begin{tabular}{|c|c|c|c|c|c|c|c|}
\hline \multirow[t]{2}{*}{ Pathway } & \multirow[t]{2}{*}{ Gene } & \multirow{2}{*}{$\frac{\text { Gene BF }}{\text { iBRI }}$} & \multirow[t]{2}{*}{ Likely assoc. variant } & \multicolumn{3}{|l|}{$\mathrm{p}$ value } & \multirow{2}{*}{$\frac{\text { Gene BF }}{\text { BRI }}$} \\
\hline & & & & C-alpha & weighted sum & comp. step-up & \\
\hline \multirow{3}{*}{$\begin{array}{l}\text { NER } \\
(\mathrm{BF}=30.8)\end{array}$} & XPC & 180.1 & exm292542 & 0.006 & 0.164 & 0.588 & 7.2 \\
\hline & ERCC6 & 6.6 & exm824160 & 0.374 & 0.337 & 0.78 & 1.0 \\
\hline & ERCC8 & 4.4 & exm456709 & 0.386 & 0.032 & 0.077 & 1.5 \\
\hline \multirow{2}{*}{$\begin{array}{l}\text { NHEJ } \\
(\mathrm{BF}=11.0)\end{array}$} & PRKDC & 19.3 & exm699583 & 0.084 & 0.079 & 0.083 & 1.6 \\
\hline & NHEJ1 & 5.3 & exm 267278 & 0.404 & 0.120 & 0.327 & 1.1 \\
\hline \multirow{3}{*}{$\begin{array}{l}\text { XLR } \\
(B F=9.7)\end{array}$} & DCLRE1B & 46.4 & exm85671 & 0.079 & 0.020 & 0.012 & 7.3 \\
\hline & POLN & 31.4 & exm382361 & 0.089 & 0.024 & 0.017 & 4.8 \\
\hline & DCLRE1A & 2.6 & exm857026 & 0.666 & 0.405 & 0.780 & 0.6 \\
\hline \multirow{2}{*}{$\begin{array}{l}\mathrm{ATM} \\
(\mathrm{BF}=2.2)\end{array}$} & $\mathrm{MDC1}$ & 6.8 & exm528405 & 0.359 & 0.100 & 0.113 & 2.0 \\
\hline & ATM & 4.6 & exm953816 & 0.37 & 0.174 & 0.274 & 1.7 \\
\hline
\end{tabular}

Genes are organized based on the DNA repair pathway that the genes are involved in. The pathway BFs are reported under each pathway. Likely assoc. variant $=$ The most likely associated variant within the gene determined based on marginal BFs; comp. $=$ comprehensive. Genes in bold have been detected in an independent study.

gene's $\mathrm{p}$ values for $\mathrm{C}$-alpha, for weighted sum, and for the comprehensive step-up approach, and (4) the gene's BF under BRI. Within table 2 we can see that four specific DNA repair pathways have strong evidence of an association with colon cancer: (1) nucleotide excision repair (NER), (2) non-homologous end joining (NHEJ), (3) cross-link repair (XLR), and (4) ataxia telangiectasia mutated (ATM). The most likely DNA repair pathway associated with colon cancer is NER with a pathway BF of 30.8 and a corresponding $\alpha$ of 0.31 (95\% CI -1.81 to 1.86 ). Within NER, genes XPN, ERCC6, and ERCC8 have strong evidence of an association. These genes have also been detected as being associated with colon cancer in an independent data set. However, both ERCC6 and ERCC8 would not have been detected using the non-integrative alternative methods.

\section{Discussion}

The original BRI has many potential advantages over alternative rare variant approaches. The approach selects a subset of variants to include in the risk index, allows for both risk and protective variants to contribute, and formally incorporates the uncertainly in that selection. This allows for increased power when the number of causal variants is small in proportion to the overall number of variants evaluated and avoids dilution of power if both risk and protective variants are present. Additionally, the BRI allows for formal inference via BFs for both regional association and for the contribution of any specific variant. For the iBRI presented here, we make two important extensions. First, within a single regression analysis model, we include a multi-regional profile consisting of a risk index for each region. Second, we allow for prior information to guide the inclusion of each region and the inclusion of which variants within each regional risk index.

As genetic association studies move forward to acquire numerous rare variants across the genome using array or sequencing technologies, the ability to model multiple regions will be crucial for combined analyses. A regression framework allows this to be accomplished by simply expanding the linear predictor to include a sum over all region-specific risk indexes. Such an extension is not easily implemented with some alternative approaches, such as C-alpha [8], in which the analysis is either on a specific region independently or across numerous regions in aggregate. However, even though such an extension is conceptually straightforward in regression, implementation to numerous regions quickly leads to limitations in the number of regions that can be feasibly evaluated. Conventional model selection procedures could be employed on a set of regional risk indexes, but this would require pre-constructing the indexes and a loss of the ability to have variant selection within each regions. Furthermore, since uncertainty in the final model determination is not 
incorporated in many of these approaches, problems can arise with hypothesis testing, such as an increase in type I error for forward-selection algorithms.

BMU approaches offer a solution that allows for the uncertainty in the model search to be propagated up from selection of variants within each region to selection of regions across all variants evaluated. In addition, formal and valid inference can be accomplished via BFs. Specifically in terms of formal inference, iBRI allows for multilevel inference at the global, regional and marginal levels. This intuitive multi-level inference provides a practitioner with the ability to not only assess the global impact of rare variation, but to also pinpoint the association within pathways, genes, and specific variants within these regions. Thus, with limited resources for follow-up studies, the practitioner can focus more attention on smaller regions surrounding specific variants rather than entire regions. Conversely, inference on higher-level combinations allows for subtle effects from variants, regions and genes to guide broad conclusions regarding pathways and their potential aggregate effect. In the analysis of the DNA repair pathway, a single $\mathrm{BF}$ of 30.8 indicates a noteworthy association for the NER pathway in aggregate (table 2) formally summarizing the overall evidence from three genes with elevated BFs greater than 3 (XPC, ERCC6, and $E R C C 8)$ and seven genes with nominal BFs (DDB2, ERCC2, ERCC3, ERCC4, ERCC5, RAD23A, and XAB2).

Another key feature of the iBRI approach is the integration of prior biological information. Due to the difficulty for marginal rare variant analysis and rare variant load tests to indicate specific variants, many investigators are simply filtering variants based on biofeatures and annotation. Unfortunately, these often reduce to ad hoc deterministic decisions based largely on the whim of the investigator. The inclusion of prior covariates in a secondstage probit regression on the probability of inclusion of any variant allows the investigator to focus on characterizing the variants, using information such as functional prediction scores or pathway level information (PolyPhen-2 [19], SIFT [20], MutationTaster [21], LRT [22], PANTHER [23], PhD-SNP [24], SNPs3D [25], PMut [26], SNAP [27], MutPred [28], and SNPs\&GO [29]). Moreover, these prior covariates simply define an exchangeable class of variants and do not predetermine the weight or importance of the information a priori [30-33]. The impact on probability of inclusion is estimated from the data. This is clearly seen in the WECARE Study example for BRCA1 (table 1). A priori, variants' biological effects were determined as either truncation, missense, IVS, or synonymous. Upon analysis, since numerous

Integrated Bayesian Risk Index truncation variants demonstrate evidence for association, those truncation variants with more modest numbers were deemed more noteworthy. For example, based simply upon the case-control comparison (2 cases vs. 0 controls), variants V52, V3, V112, and V106 have BFs of approximately 13 from the BRI analysis. Rather than offering post hoc additional justification of that, these variants are of interest because they are simply truncation variants, the iBRI formally incorporates that information, estimates its impact, and elevates the BF for these variants to over 600 . Additionally, since the impact of prior information is estimated from the data, if that information is non-informative, our simulation results indicate that there is no increase in the detection of false positives (fig. 1,2). Conversely, as the prior information becomes more informative in terms of specificity and sensitivity in identifying the true causal variants, the estimated as increase and performance is improved.

The BRI and iBRI methods do come with an added computational cost of running a $\mathrm{MH}$ algorithm to sample models from the high-dimensional model space as well as a Gibbs algorithm to sample posterior estimates of the effects of the variant-specific external biological covariates, $\alpha$ in the case of iBRI. The computational complexity of running one iteration of the $\mathrm{MH}$ algorithm corresponds to the computational cost of estimating model-specific parameters and the marginal likelihoods for each unique model sampled. This scales linearly with the sample size $n$ and cubically with respect to the number of model-specific parameters being estimated. Thus, an increase in sample size will not cause a significant increase in computation time of the $\mathrm{MH}$ algorithm. However, as the number of region-specific risk indices being incorporated within any given model increases, the computation time of the algorithm will increase substantially. The computational complexity of one iteration of the Gibbs algorithm under the iBRI approach scales linearly with respect to the number of variant-specific external covariates, $c$, and the total number of variants under consideration, $p$. Therefore, as we increase these parameters, we will not see a significant increase in computation time per iteration of the Gibbs sampling algorithm. With regards to the added computational complexity of iBRI for analyzing multiple regions versus modeling a single region, the analysis of 1 gene region for the WECARE Study took approximately $3 \mathrm{~h}$ for 100,000 iterations on a $2.3 \mathrm{GHz} \mathrm{CPU}$, whereas the analysis of 114 gene regions for the KY data took approximately $6 \mathrm{~h}$ for the same number of iterations on the same machine. Thus, we see that the computation time to run iBRI on a study involving multiple regions doubles with respect 
to running the same approach on a single region; most likely demonstrating the added computational complexity of estimating effects of multiple risk indices for each unique model. With regards to the added computational complexity of the Gibbs sampling algorithm needed for the iBRI approach versus the BRI approach, the analysis of 134 rare variants within the WECARE Study took approximately $2.5 \mathrm{~h}$ for 100,000 iterations of the $\mathrm{MH}$ algorithm under the BRI approach compared to $3 \mathrm{~h}$ for 100,000 iterations of the Gibbs/MH algorithm under the iBRI approach. This increase of $0.5 \mathrm{~h}$ to run the iBRI approach compared to the BRI approach on the WECARE Study demonstrates the added computational time required to perform the Gibbs algorithm of iBRI.

These examples give the user a good idea of the computational complexity of the BRI and iBRI approaches for a set number of iterations of the Gibbs/MH algorithms. However, it needs to be noted that as the total number of variants of interest increases, the total number of iterations of the algorithms needed for convergence of posterior quantities will also increase. Currently, we suggest doing two independent runs of the search algorithms and comparing the global and marginal posterior quantities computed under a set number of iterations of each independent run to determine if the algorithm has converged. While we have shown that the integration of external variant-specific covariates into the probability that each variant is associated can increase the efficiency of the model search algorithm (fig. 3) when the covariates are truly informative with regards to the causal variants, it is of future interest to investigate alternative algorithms and computational techniques to allow for whole-genome analysis. Also, as the number of variant-specific covariates increases, there is the potential for an additional level of uncertainty into which variant-specific covariates should be used to inform the variant and region selection.

In conclusion, the iBRI method is a powerful and flexible regression framework that allows for the investigation of rare variants across numerous regions and the integration of prior biological annotation and functional information. In addition, adjustment variables, such as principle components for population structure, can be included as well as an extension to non-dichotomous outcome traits such as quantitative or survival traits.

\section{Acknowledgements}

This work has been partially supported by the National Institute ofHealth (grants R01ES016813,U01DA020830, R21HL115606 and R01CA14561). We thank the WECARE Study Collaborative Group (R01 CA097397 and U01 CA083178) and the Kentucky Colorectal Cancer Study (U54 CA116867 and K22 CA120545) for the example data.

\section{Web Resources}

URL for the R BVS package is: http://cran.r-project.org/web/ packages/BVS/index.html.

\section{References}

1 Morgenthaler S, Thilly WG: A strategy to discover genes that carry multi-allelic or monoallelic risk for common diseases: a cohort allelic sums test (CAST). Mutat Res 2007;615: $28-56$.

2 Li B, Leal SM: Methods for detecting associations with rare variants for common diseases: application to analysis of sequence data. Am J Hum Genet 2008;83:311-321.

-3 Madsen BE, Browning SR: A groupwise association test for rare mutations using a weighted sum statistic. PLoS Genet 2009;5:e1000384.

4 Hoffmann TJ, Marini NJ, Witte JS: Comprehensive approach to analyzing rare genetic variants. PLoS One 2010;5:e13584.

5 Quintana MA, Berstein JL, Thomas DC, Conti DV: Incorporating model uncertainty in detecting rare variants: the Bayesian risk index. Genet Epidemiol 2011;35:638-649.
6 6 Bhatia G, Bansal V, Harismendy O, Schork NJ, Topol EJ, Frazer K, et al: A covering method for detecting genetic associations between rare variants and common phenotypes. PLoS Comput Biol 2010;6:e1000954.

7 Price AL, Kryukov GV, de Bakker PIW, Purcell SM, Staples J, Wei LJ, et al: Pooled association tests for rare variants in exon-resequencing studies. Am J Hum Genet 2010;86: 832-838.

8 Neale BM, Rivas MA, Voight BF, Altshuler D, Devlin B, Orho-Melander M, et al: Testing for an unusual distribution of rare variants. PLoS Genet 2011;7:e1001322.

9 Cardin NJ, Mefford JA, Witte JS: Joint association testing of common and rare genetic variants using hierarchical modeling. Genet Epidemiol 2012;36:642-651.

-10 Wilson MA, Iversen ES, Clyde MA, Schmidler SC, Schildkraut JM: Bayesian model search and multilevel inference for SNP association studies. Ann Appl Stat 2010;4:1342-1364.
11 Clyde M, George EI: Model uncertainty. Statist Sci 2004;19:81-94.

12 Hoeting JA, Madigan D, Raftery AE, Volinsky CT: Bayesian model averaging: a tutorial (with discussion). Stat Sci 1999;14:382-401.

13 Quintana MA: BVS: Bayesian variant selection: Bayesian model uncertainty techniques for genetic association studies. http://cran.rproject.org/web/packages/BVS/index.html.

14 Bernstein JL, Langholz B, Haile RW, Bernstein L, Thomas DC, Stovall M, et al: Study design: evaluating gene-environment interactions in the etiology of breast cancer - the WECARE Study. Breast Cancer Res 2004; 6:R199-R214.

$\checkmark 15$ Begg CB, Haile RW, Borg A, Malone KE, Concannon P, Thomas DC, et al: Variation of breast cancer risk among BRCA1/2 carriers. JAMA 2008;299:194-201. 
16 Borg A, Haile RW, Malone KE, Capanu M, Diep A, Törngren T, et al: Characterization of BRCA1 and BRCA2 deleterious mutations and variants of unknown clinical significance in unilateral and bilateral breast cancer: the WECARE Study. Hum Mutat 2010;31:E1200E1240.

17 Malone KE, Begg CB, Haile RW, Borg Å, Concannon P, Tellhed L, et al: Populationbased study of the risk of second primary contralateral breast cancer associated with carrying a mutation in BRCA1 or BRCA2. J Clin Oncol 2010;28:2404-2410.

18 Li L, Plummer SJ, Thompson CL, Merkulova A, Acheson LS, Tucker TC, Casey G: A common $8 \mathrm{q} 24$ variant and the risk of colon cancer: a population-based case-control study. Cancer Epidemiol Biomarkers Prev 2008; 17:339342.

19 Adzhubei IA, Schmidt S, Peshkin L, Ramensky VE, Gerasimova A, Bork P, Kondrashov AS, Sunyaev SR: A method and server for predicting damaging missense mutations. Nat Methods 2010;7:248-249.

20 Kumar P, Henikoff S, Ng PC: Predicting the effects of coding non-synonymous variants on protein function using the SIFT algorithm. Nat Protoc 2009;4:1073-1081.

-21 Schwarz JM, Rodelsperger C, Schuelke M, Seelow D: MutationTaster evaluates diseasecausing potential of sequence alterations. Nat Methods 2010;7:575-576.
22 Chun S, Fay JC: Identification of deleterious mutations within three human genomes. Genome Res 2009;19:1553-1561.

23 Thomas PD, Kejariwal A, Guo N, Mi H, Campbell MJ, Muruganujan A, LazarevaUlitsky B: Applications for protein sequencefunction evolution data: mRNA/protein expression analysis and coding SNP scoring tools. Nucleic Acids Res 2006;34(Web Server issue):W645-W650.

24 Capriotti E, Calabrese R, Casadio R: Predicting the insurgence of human genetic diseases associated to single point protein mutations with support vector machines and evolutionary information. Bioinformatics 2006;22: 2729-2734.

25 Yue P, Melamud E, Moult J: SNPs3D: candidate gene and SNP selection for association studies. BMC Bioinformatics 2006;7:166.

26 Ferrer-Costa C, Gelpi JL, Zamakola L, Parraga I, de la Cruz X, Orozco M: PMUT: a webbased tool for the annotation of pathological mutations on proteins. Bioinformatics 2005; 21:3176-3178.

27 Bromberg Y, Rost B: SNAP: predict effect of non-synonymous polymorphisms on function. Nucleic Acids Res 2007;35:3823-3835.
28 Li B, Krishnan VG, Mort ME, Xin F, Kamati KK, Cooper DN, Mooney SD, Radivojac P: Automated inference of molecular mechanisms of disease from amino acid substitutions. Bioinformatics 2009;25:2744-2750.

29 Calabrese R, Capriotti E, Fariselli P, Martelli PL, Casadio R: Functional annotations improve the predictive score of human diseaserelated mutations in proteins. Hum Mutat 2009;30:1237-1244.

30 Greenland S: Putting background information about relative risks into conjugate prior distributions. Biometrics 2001;57:663-670.

31 Conti DV, Witte JS: Hierarchical modeling of linkage disequilibrium: genetic structure and spatial relations. Am J Hum Genet 2003;72: 351-363.

32 Conti D, Lewinger J, Swan G, Tyndale R, Benowitz N, Thomas P, Swan G: Using ontologies in hierarchical modeling of genes and exposures in biologic pathways; National Cancer Institute. Phenotypes and Endophenotypes: Foundations for Genetic Studies of Nicotine Use and Dependence. Tobacco Control Monograph No. 20. Bethesda, US Department of Health and Human Services, National Institutes of Health, National Cancer Institute. NIH Publication No. 09-6366, August 2009.

33 Wilson MA, Baurley JW, Thomas DC, Conti DV: Complex system approaches to genetic analysis Bayesian approaches. Adv Genet 2010;72:47-71. 